\title{
Le sacrifice consenti à regret
}

Aperçu sur le sacrifice chez les Santal de l'Orissa (Inde)

Sacrifices consented reluctantly: A glimpse of sacrifice among the Santal in

Orissa (India)

\section{Marine Carrin-Bouez}

\section{OpenEdition \\ Journals}

Édition électronique

URL : http://journals.openedition.org/span/391

DOI : 10.4000/span.391

ISSN : 2268-1558

Éditeur

École pratique des hautes études. Sciences humaines

\section{Édition imprimée}

Date de publication : 1 septembre 1978

Pagination : 135-149

ISSN : 0294-7080

Référence électronique

Marine Carrin-Bouez, "Le sacrifice consenti à regret », Systèmes de pensée en Afrique noire [En ligne], 3 | 1978, mis en ligne le 04 juin 2013, consulté le 01 mai 2019. URL : http://journals.openedition.org/ span/391 ; DOI : 10.4000/span.391 


\section{LE SACR I F I C E C O N S E N T I A REGRET Aperçu sur le sacrifice chez les Santal de l'Orissa (Inde). par Marine Carrin-Bouez}

Le sacrifice occupe chez les Santal (1) une place tout aussi centrale que dans la société hindoue, mais à des titres différents. Dans 1a société des castes, il apparâ̂t comme un principe structurant de la sociêté globale, puisqu'il sous-tend l'ordre socio-cosmique (dharma) qui préside à la hiérarchie fonctionnelle des vama (2). Chez les Santal, en revanche, on ne peut l'appréhender selon un modèle unique dont les principes structureraient l'ensemble de la société, mais selon plusieurs modèles qui renvoient à une idéologie ambiguë du sacrifice.

Certains aspects du sacrifice évoquent, certes, les rites de 1 'hindouisme populaire: les Santal participent, aux côtês des castes qui vivent dans leur voisinage, à des fêtes hindoues, principalement les fêtes des déesses ; ces fêtes ne les concernant pas au même

1. Les Santal sont un groupe austro-asiatique indien comprenant plus de trois millions de personnes réparties dans divers Etats du Bihar, du Bengale et de 1'Orissa. Ils forment une unité endogame qui se situe en marge du système des castes. L'hindouisme constitue cependant pour eux un pôle de rêférence certain, avec cette réserve toutefois que les emprunts à 1 'hindouisme sont réinterprétés par le discours religieux santal.

Le système de transcription des termes santal suivra ici P. 0 . Bodding (1929-1936); pour les termes sanskrit, on adoptera les conventions du dictionnaire sanskrit-français de N. Stchoupak, L. Nitti et L. Renou, 1959, Maisonneuve, Paris.

2. varma : "catégorie", "ētat". Ce terme désigne plus précisément 
titre que les sacrifices qu'ils adressent à leur bonga (3) : ils vont voir (h́le l) les fêtes hindoues, mais n'y participent pas.

Le mythe de création santal évoque les premières libations de bière de riz que le couple ancestral a répandu sur le sol pour marán buru, la divinité suprême du panthéon santal. Aujourd'hui encore, l'aîné de la maison (orak') 1'offre à ses ancêtres dans l'autel ancestral. Elle constitue 1 'offrande minimale que les premiers germains consoment par erreur puisqu'elle êtait destinée aux divinitês dans le mythe de création, ceci les entraîne à commettre l'inceste et à procréer l'humanité (les Santal).

$$
\begin{gathered}
\dot{I} \quad L A \quad P R O M E S S E \quad D E \quad S A C R I F I C E \\
E T S L E S \quad S A C R I F I A N T S
\end{gathered}
$$

1. Les témoins du sacrifice.

Les Santal affirment que les hommes ne peuvent deviner ce que veulent les divinités, ils insistent toutefois sur 1'intention, et la disponibilité du sacrifiant au sacrifice. Le sacrifiant est ici celui au bénéfice de qui le rite est accomplì et renvoie à une unité de culte : village, quartier, lignée, maison ou individu. Tout sacrifice peut être annoncé par une promesse de sacrifice qui est elle-même un sacrifice.

La promesse du sacrifice se rapporte à la certitude de l'efficacitê du rite et évoque la notion hindoue de śradaha (confiance) telle qu'elle apparaît dans le sacrifice védique : "(..) la śraḋhā, avant d'être la "foi", est la confiance que le sacrifiant a en l'efficacité

chacune des catëgories fonctionnelles (prêtre, guerrier, agriculteur, serviteur) autour desquelles s'organise la société hindoue de 1 'Inde ancienne.

3. Le terme bonga, utilisé comme radical nominal, est le terme générique pour désigner une divinité ; comme radical verbal, il prend les désinences propres à n'importe quel verbe et signifie "sacrifier". 
du rite qu'il entreprend, et surtout dans la compétence du rotvij (4) qu'il choisit pour officier dans ce rite." (Ch. Malamoud, 1976, p. 161) (5).

Cette intention du sacrifiant est manifeste dans la "promesse de sacrifice" (sim agom, litt. "promesse de volaille"). La promesse d'un sacrifice consiste à différer 1 'immolation de la victime qui est parfois consacrée à la divinité. Dans la promesse de sacrifice,le sacrifiant considère que sa parole devient matière oblatoire. La "promesse" est faite par le prêtre -- ou le sacrifiant -- devant une divinité "têmoin" (saket') (6). Elle est adressée à la divinité à qui le futur sacrifice est destiné.

2. Purification et omissions rituelzes.

L'état de pureté est requis pour le sacrifiant ; cet état s'acquiert en respectant les interdits sexuels et alimentaires la veille des sacrifices. Un bain précède généralement le sacrifice. La "purification" (um) (7) donne "un pouvoir" au sacrifice; en revanche, un tel état de pureté n'est pas requis pour l'exorcisme qui fonctionne sur une logique du "même", où l'impur chasse l'impur, le désordre la confusion. On peut également être en état d'impuretê pour sacrifier à une divinité malveillante, souvent cannibale. Certains mythes d'origine de sous-

4. Nom donné à une catêgorie d'officiant vêdique.

5. La "promesse d'une volaille" a lieu surtout dans les rites de cure. On demande à la divinité de montrer elle aussi des signes de sa bienveillance en améliorant l'état du malade. Des implications économiques pèsent sur ce type de prescription, car, faute de volaille, on en promet une. En revanche, lorsque le sacrifiant dispose d'une volaille et diffère le sacrifice, le prêtre fait une marque à celle-ci en lui coupant un ongle pour être sûr de la reconnaître parmi d'autres plus tard.

6. La divinité tẹmoin est souvent un ascète hindou divinisé, le muni, "sage", ou marán bum lui-même. Dans le cas de témoins hindous, on remarque que ceux-ci cautioment par leur appartenance à la religion dominante, 1 'engagenent que les Santal prennent vis-à-vis des divinités.

7. La notion santal d'impureté est davantage liée au danger que dans l'hindouisme; les Santal disent toutefois que c'est par protection rituelle du brahmane que la purification peut s'opérer. Il s'agit ici d'une reprise idéologique du discours hindou puisque les brahmanes ne vont pas officier dans les villages santal. 
clan expliquent souvent la scission d'un clan en sous-clan par une négligence d'ordre rituel. Ainsi, dans le mythe d'origine, les gens du sous-clan "vermillon" (8) accomplissent leurs sacrifices avec tous les ingrédients nécessaires, tandis que les gens du sous-clan "simple" (sada) ont oublié le vermillon et n'utilisent, pour marquer leur victime et tracer certains points dans l'aire rituelle, que de la farine de riz (holon); c'est pourquoi on dit qu'ils accomplissent le rite de façon "simple" (sada), d'où leur nom. Ainsi, ceux qui se sont trouvés dépourvus de vermilion n'ont pas disposé de tous les ingrêdients rituels nécessaires au bon accomplissement du sacrifice. Omettre un de ces éléments constitue une faute rituelle qui devient une norme pour le groupe. L'erreur rituelle peut aussi porter sur la manière de sacrifier, soit qu'on ne respecte pas les interdits sexuels et alimentaires préalables à tout sacrifice, soit qu'on se révèle d'une façon ou d'une autre incapable de sacrifier ; ainsi, le "pouvoir de la bouche" peut faire dêfaut à un prêtre qui ne prononce pas correctement les invocations rituelles.

3. Les femmes exclues du sacrifice.

Une incapacité permanente à sacrifier est attribuée aux femmes santal qui se trouvent exclues du sacrifice; la possession leur est également interdite. Un mythe recueilli par P. 0. Bodding (1925, p. 228), intitulé "les femmes stupides", fournit toutes les justifications de 1 'incapacité des femmes à sacrifier. Dans ce mythe, les hommes ont quitté le village pour aller au marché et ont demandé aux femmes de préparer la bière de riz nécessaire à la célébration du sacrifice d'un boeuf (dángra) à leur bonga de lieu (dont le nom est transinis par le père à son fils aîné). Les hommes ne revenant pas, les femmes décident de sacrifier elles-mêmes à la divinité de lieu ; toujours dans ce mythe, les belles-soeurs se réunissent et consultent 1 'aînée d'entre elles qui connaît le type de victime destiné à cette divinitê, soit un boeuf non

8. Ce sous-clan est présent dans tous les clans, on le qualifie parfois de $n i j$ ("pur", "originel"). Le langage du sacrifice permet de différencier les sous-clans, à l'intérieur des clans. 
castré, et les invocations que les hommes de leur lignée ont coutume de prononcer au moment du sacrifice. Les femmes préparent alors l'aire rituelle à l'aide de bouse de vache, puis chacune demande à sa voisine de mettre à mort le boeuf ; celui-ci finit par s'enfuir et les divinités en concluent qu'elles ne peuvent rien recevoir de la main des femmes. Au début de ce mythe, les femmes avaient, de fait, pressenti leur inaptitude au sacrifice, puisqu'elles ont dit aux hommes qu'elles ne sauraient en aucun cas couper en morceaux la chair de la victime. Mise à mort et morcellement s'opposent ensemble à l'idée de reproduction conçue comme un modèle cyclique de pêriodicité à l'intérieur duquel les femmes ont un rôle majeur, celui de reproduire la lignée (9). En revanche, les femmes offrent régulièrement de la bière de riz dans 1'autel ancestral (bhitri) de leur mari lors des fêtes agraires. Cette offrande cérémonielle peut être publique, c'est le cas pour la fête du bétail (octobre-novembre), sohrai. Les femmes présentent aux boeufs, attachés à des piliers érigés à cette intention dans la rue principale du village, une offrande d'herbe sacrée ; cette offrande est déposée dans un van à riz que les femmes font tourner autour des têtes des boeufs ; ce type d'offrande végétale est répété au cours des principales fêtes, on l'appelle cumaran, le "baiser cérémoniel". Il s'agit d'une pratique rituelle qui n'est pas considérée comme un sacrifice. De ce fait, elle est permise aux femmes. Le baiser cérémoniel n'est accompagné d'aucune invocation à la différence de l'offrande végétale consentie à une divinité bienveillante qui, elle, est considérée comme un sacrifice (les Santal 1'appellent bonga) si elle est accompagnée d'une invocation rituelle, et êventuellement d'une libation de bière de riz; ce dernier ingrédient, par son caractère fermenté, se situe à mi-chemin des offrandes carnées et végétales. D'un point de vue plus général, le sacrifice se définit, pour les Santal, par les trois aspects complémentaires suivants : libation de sang, invocation, partage de la chair et du sang du sacrifice; la contradiction apparente tient ici au statut ambigu de la libation de sang dans la définition : elle n'est pas un

9. Du point de vue symbolique, les femmes reprêsentent chez les Santa1 la régénération de la lignée, tandis que les hommes en assurent la continuité. 
êlément de celle-ci, mais permet d'augmenter 1'efficacité escomptée du sacrifice.

L'interdit de sacrifice qui frappe les femmes s'explique par la suspicion de sorcellerie dont elles font 1 'objet, qui les situe à michemin du monde des humains et de celui des divinités. Les femmes santal ne participent au sacrifice qu'en tant qu'épouses et auxiliaires des sacrifiants. La présence de l'épouse du prêtre du village, la naeke era, a toutefois une importance du point de vue du rite car elle représente, avec son mari, le couple ancestral sur terre. Pour cette raison, on dit encore "qu'elle ne peut pas être une sorcière". A la différence des autres femmes, elle peut pénétrer dans le bosquet sacré du village et préparer la bière de riz destinée aux libations des sacrifices publics. Sa présence est enfin requise pour la fondation d'un bosquet sacré dans le village (10). Les Santal rejettent les femmes du côté de la sorcellerie pour affirmer en même temps que l'êpouse d'un prêtre est nécessaire à la fondation du lieu de culte le plus important du village, le bosquet sacré.

$$
I I . \quad L{ }^{\prime} I D E ́ O L O G I E \quad D U \quad S A C R I F I C E
$$

1. Catégories d'officiants et modèle du sacrifice.

Les invocations rituelles santal déplorent à loisir l'incapacité humaine à deviner les désirs des divinités en matière de sacrifice.

La disjonction entre sacrifiant et sacrificateur (11) est ici moins nette que dans l'hindouisme, en ce qui concerne, du moins, les

10. Le bosquet sacré est le lieu de résidence du couple ancestral sur terre; il est spécifique à chaque village.

11. Les rites qui se déroulent dans le bosquet sacré sont généralement répétés au niveau domestique dans les autels ancestraux des maisons ; chaque chef de maison est donc sacrifiant dans les rites de village puisqu'il fournit une victime destinée au sacrifice du bosquet sacré ; il est à la fois sacrifiant et sacrificateur dans les rites domestiques. 
rites célëbrés par l'ensemble du village. Les volailles et les chèvres sont recueillies, dans chaque maison, par un messager du prêtre du village. Les hommes participent aux sacrifices agraires dans le bosquet sacré, le prêtre du village immole les victimes ; il ne reçoit pas d'honoraires rituels pour cette fonction. Une terre lui est toutefois allouée généralement par l'ensemble du village, une partie du riz qui y est récolté sert à la préparation de la bière sacrificielle ; elle est préparée, à chaque fois, par l'épouse du prêtre qui l'apporte elle-même au bosquet sacré; le reste de la récolte de ce champ est réservé à l'usage personnel du prêtre et de sa maison (12).

En revanche, lorsqu'il sacrifie pour chasser une épidémie ou un fléau nature1, il reçoit en honoraires rituels un linge blanc offert par l'ensemble des villageois.

Dans les rites domestiques, sacrificateur et sacrifiant sont conjoints. Dans les rites de cure, au contraire, le sacrifiant est un individu, le malade, qui donne des honoraires rituels au ojha, "prêtredevin", qui se rend à la maison du malade pour célébrer les rites de cure.

Deux modèles du sacrifice s'organisent autour des deux types de prêtres ; 1'un est représenté par le naeke (prêtre du village) ; il peut être défini comme un modèle de reproduction de la norme et de la prospêrité du village. L'autre engage, on va le voir, la personne même du ojha, "prêtre-devin".

2. Le prêtre du vizzage et l'exactitude liturgique.

$\mathrm{Si}$, pour une raison ou une autre, le prêtre de village se trouve incapable d'immoler lui-même la victime, il peut demander à l'un de ses auxiliaires de le faire, mais il doit asperger lui-même la victime d'eau lustrale. S'il commet une erreur en récitant l'invocation rituelle, il encourt alors la colère des divinités du bosquet sacré, qui . demandent alors à Tamib bonga, le bonga tigre de la forêt de venir s'attaquer aux villageois en provoquant d'abord en eux une possession

12. Lorsqu'au moment des semailles (erok'), le prêtre de village accomplit un rite destiné à assurer la fécondité de ce champ, il augure du même coup de 1 'heureuse issue des sacrifices à venir. 
incontrôlable (mm daka), puis en les enlevant dans la forêt. Le prêtre du village doit alors s'amender auprès des divinités et invoquer 1'ivresse, par exemple, pour justifier son erreur. Il peut ainsi expliquer cet état d'ivresse en le mettant sur le compte d'une visite faite à des parents dans un autre village ou à la célébration de que1que événement important, occasions pour lesquelles il est normal de boire plus que de coutume.

Le naeke est porteur de "valeurs" dans la société santal, comme 1 'est le brahmane dans 1a société hindoue. On lui attribue une connaissance juste des fautes, que l'offrande de son propre sang lui garantit : chaque année, en effet, le naeke offre son sang à pargana boñga, divinité ancestrale des chefs de village, et demande à cette divinité de l'aider à discerner 1 'intêrêt des villageois (13).

On peut voir dans cet espect éthique de l'activité du naeke une reprise des concepts de pap (pêché) et de puniya (mérite) de 1.'hindouisme populaire. La conduite prescrite dans la société des castes est de s'efforcer d'augmenter le mërite puniya. Chez les Santal, la conduite vertueuse, juste, du naeke et de son épouse doit être conforme au modèle du couple ancestral qui vivait à l'êpoque du mythe de création, temps de la chasse et de la cueillette, antérieur aux querelles de clans.

3. Le prêtre-devin et son corps dans le sacrifice.

Le ojha offre, au contraire, son sang chaque fois que cela s'avère nécessaire au cours d'un rite de cure, et il reçoit alors du sacrifiant des honoraires rituels spêcifiques, les balkati (14), ou

13. Cette offrande de sang, buz mayam, émane d'une décision personnelle du naeke qui émet une sorte de voeu personnel envers une divinité associée aux principes politiques du village. Ce rite vise en outre à garantir l'efficacité symbolique de sa personne, qui se manifeste particulièrement au cours des rites d'invocation de la pluie, où le naeke apparaît comme un "faiseur de pluie", parce qu'il représente avec son épouse le couple ancestral.

14. Il s'agit des balkati, prix du sang; ces honoraires sont versés par le malade. La relation qui unit ici le prêtre et les sacrifiants (le malade) se traduit dans une logique des prestations. Ceci n'est pas sans évoquer 1 'hindouisme; comme le remarque $\mathrm{Ch}$. Malamoud : "Dans l'Inde, plus clairement qu'ailleurs peut-être, la relation liturgique ainsi comprise dans sa complexité aura été le modèle de toute relation entre employeur et employé, plus précisément entre utilisateur et fournisseur de services." (1976, p. 157). 
honoraires pour le sang versé. Le ojha associe l'offrande de sang, bul mayam, à la possession (mom). En effet, par l'offrande de sang comme par la possession, il met son propre corps en contact avec les divinités.

Le "prêtre-devin" offre son sang à partir de cinquante-cinq points symboliques localisés dans son corps ; ces points ne sont pas nommés, ils sont le plus souvent situés aux articulations. Il réalise ainsi un morcellement de son propre corps qui inverse celui de sa propre initiation, au cours de laquelle son corps, d'abord démembré symboliquement, a été reconstitué par son maître. Lors de la cure, le ojha totalise en un point le mal qui accable le malade et 1 'extirpe par morsure.

D'après 1 'exégèse que nous fournissent les ojha eux-mêmes, l'offrande de sang, appelée "sang menstruel", sitka mayam (15), est ici le substitut du sacrifice humain ; elle est d'une efficacité symbolique supérieure à celle des autres victimes, dans la mesure où elle apaise les divinités les plus cannibales, mais elle entraîne en contre-partie pour le ojha une dêperdition de sa force spirituelle, jiu, qu'il est seul capable de recouvrer ensuite.

4. : La prescription mituelle.

Celle-ci est plus ou moins fixe selon l'unité de culte considérée, divinitês carnivores et végétariennes s'opposant moins nettement que dans le panthéon hindou. Les prêtres santal admettent généralement qu'il $n^{\prime} y$ a pas adéquation entre le désir de sacrifice que les humains prêtent aux divinitês et l'efficacité attendue du sacrifice. Les invocations rituelles qu'ils récitent esquissent un parallèle entre le monde des divinités et celui des humains, en disant que, tandis que les hommes mangent sur terre, les divinités mangent dans l'aire rituelle (16).

Le monde des divinités est donc la contre-partie de celui des humains mais cette homologie soutient une autre équivalence, celle du

15. Au cours du voyage rituel qui précède son initiation de ojha, le disciple porte un vêtement féminin ; en tant qu'initié, il est considéré comme la "fiancée" de son mâ̂tre. Sur ce point, cf. M. Carrin-Bouez, L'ascension des fillandres, Cahiers de littérature orale, 3, pp. 89-117.

16. L'aire rituelle est simplement délimitée par l'officiant qui purifie le sol à 1 'aide de bouse de vache. 
sacrifice et de la mort : "Les hommes meurent comme des victimes sacrificielles, que tout soit bien ainsi".

Ce type assez courant d'assertion nous suggère 1 'existence, chez les Santal, d'une pensée "négative" du sacrifice dont il faut sans doute chercher les raisons dans cet "envers" du sacrifice que constitue I'exorcisme ou "chasse" au divinitês (husit') (17).

Certains. rites fastes concernent particulièrement 1'individu, ils sont toujours destinés à une divinitê végêtarienne et légitiment l'entrée de cet individu dans une fonction religieuse oụ politique. Ces rites de légitimation sont considérés comme des rites purs, nirza, en eux-mêmes : le sacrifiant et sa famille y reçoivent des marques d'honneur (ablution des pieds) de la part des autres villageois. Sans entrer dans le détail du rite, soulignons que la pureté qui s'attache à ce rite ne tient pas uniquement à son caractère faste et vêgétarien, mais aussi à la récitation religieuse qui l'accompagne et qui reprend sur bien des points des principes religieux liês à la notion de destin personnel, karma; le caractère pur du rite est ici marqué par une offrande de beurre clarifié et de douze sortes d'essences végêtales.

Son caractère pur est également marqué par un interdit de parole qui dure une nuit. D'une manière générale, le bruit rituel ponctue des rites destinês à des divinités carnivores comme celles de la chasse. Les interdits sexuels dominent les rites destinés à des divinités végêtariennes où le silence a sa place, 1'absence d'interdit et la simulation rituelle de 1 'acte sexuel peut marquer, au contraire, des rites destinés à des divinités carnivores pourvoyeuses de gibier.

Les sacrifices comportant une offrande de sang sont les plus nombreux. Ils ont lieu dans des situations très diverses. On distinguera

17. I1 s'agit de pratiques rituelles mettant en jeu à la fois le symbolisme d'objets par lesquels on "chasse" les divinités : van à riz, balai "perceur", et de pratiques rituelles où les incantations récitées admonestent les divinités mises en cause. Au cours des rites de cure, le prêtre-devin apaise d'abord par un sacrifice la divinité responsable des maux, puis la capture dans un pot de terre pour 1a reconduire ensuite en forêt, séjour des divinités malveillantes. Les prêtres-devins définissent 1'exorcisme comme la "chasse aux divinités malveillantes". Alors que le sacrifice implique une purification préalable, l'exorcisme requiert l'impureté : dans les rites d'exorcisme, on peut aussi sacrifier, mais en étant impur, à des divinitês elles-mêmes impures. 
les libations et sacrifices sanglants qu'offrent régulièrement les descendants d'un ancêtre commun soit à ce dernier, soit à des divinités communes de "lieu" ou de "maison", des sacrifices offerts lors des rites agraires. Les premiers, en effet, insistent sur la relation d'un groupe cultuel à un territoire donné ; le sacrifice a lieu dans un champ en friche (tondi) et rappelle l'installation de cet ancêtre au village ; le sacrifiant est alors 1 'aîné du groupe cultuel qui est généralement formé sur le groupe local de sous-clan.

Les prescriptions rituelles concernant les victimes sacrificielles des rites célébrés par le sous-clan renvoient au mythe d'origine de ce groupe et sont transmises avec le nom des divinités par le père à son fils, elles varient d'un groupe à I'autre, qui se différencie alors de son voisin par sa manière de mettre à mort la victime ou les ingrédients rituels qu'il utilise.

Les prescriptions en matière de sacrifice sont relativement fixes pour les rites de village. La couleur intervient le plus souvent come variable (18). On offre, par exemple, toujours des volailles blanches à Maran Bum, divinitê bienveillante à qui est attribuée la création du premier couple humain. Les sacrifices d'animaux roux ou de volailles aux plumes rouges caractérisent les rites de moisson ou, plus généralement, de prospérité. Les animaux noirs, enfin, sont généralement destinés aux divinités responsables de la maladie.

18. La prescription de la couleur et du sexe des volailles sacrificielles est plus complexe dans les rites de cure où la prescription elle-même devient objet de la spéculation des prêtres-devins. Sur ce point, cf. M. Carrin-Bouez, La couleur prescrite : discrimination et accumulation dans le sacrifice santal (Inde), à paraître dans un ouvrage collectif sur les couleurs, sous 1a direction de S. Tornay. 


III. LES SÉQUENCES DU RITE

Les séquences rituelles que nous présentons ici respectent 1 'ordre dans lequel elles nous ont été données ; cet ordre correspond au déroulement du rite.

- guri katen : nettoyer une aire pour le sacrifice avec de la bouse de vache; ceci se fait de la main droite de préférence, considérée comme la main pure.

-- holón khond : tracer les limites de l'aire rituelle, puis dessiner à même le sol une figure plus ou moins géométrique avec de la farine de riz. Ces figures représentent à la fois la scène où se déroule le combat des divinités bienveillantes et malveillantes et le lieu du partage des offrandes rituelles. C'est à 1'intérieur de ces figures que sont conviées les divinitês. La notion de khond se rapporte parfois à ce que l'on désigne par unité de culte : les Santal disent que les membres d'un même culte local -- de sous-clan -- qui sacrifient à la même divinité de lieu peuvent manger à partir d'une même khond, c'està-dire consommer leur part de nourriture sacrificielle.

- sindur. tikta : mettre à l'intérieur des cases du diagramme des points de vermillon dont le nombre et la rëpartition varient selon les sacrifices. Les Santal sont ici conscients d'avoir adopté un usage hindou, le vermillon, les fleurs, ou les fumigations de résine, sont censês plaire aux divinités.

Le sous-clan aîné murmu, dont les membres étaient traditionnellement les prêtres de village (naeke), se distingue : des autres murmu par le fait qu'il utilise le vermillon dans ses rituels. Le nombre et la répartition de ces points sont considérés comme une écriture. Ecrire (ol) les punji signifie pour les Santal : accumuler des tas de points "destinés aux divinités". Il s'agit, le plus souvent, de deux, trois ou quatre points qui reprêsentent une divinité. Nous n'avons pas observé de correspondance entre le nombre de points dessinés et le sexe des divinités (19).

19. Certains points ne sont pas nommés au début du rite; le prêtre se réserve ainsi la possibilité d'attribuer, au cours du rite, un nom de divinité correspondant à ces points. L"écriture" des points révèle une conception formelle de l'espace rituel qui se substitue ici à l'absence de représentation anthropomorphique des divinités qui sont seulement représentées par leur nom. 
-- amo cowal : 1'offrande de riz rituel ; ce riz est décortiqué après avoir été passé à l'eau bouillante, sans être bouilli, puis séché au soleil. La victime sacrificielle, avant d'être consacrée, doit goûter ce riz. Ceci signifie que les divinités ont accepté le sacrifice.

-- dak' timpi : consécration de la victime; 1a consécration de la victime est la même dans tous les rites. La victime est présentée à $1 a$ divinité; le prêtre lui fait une marque de vermillon au front, et lui offre du riz sacrificiel, après l'avoir aspergée d'eau lustrale. L'officiant présente le riz à la victime et guette l'assentiment des divinités. La formule santal : atan, telan, "reçu et acceptê", exprime assez clairement le caractère d'échange du sacrifice. On dit encore que le prêtre fait hatin avec la victime ; ce dernier terme signifie "diviser", "séparer" et montre que la victime instaure ici le partage, tout en étant elle-même partagée entre les divinités et les humains.

La mise à mort de la victime n'est pas désignée par un terme génêrique. Si sacrifier comprend pour les Santal un sacrifice sanglant, il semble qu'ils insistent davantage sur la libation de sang que sur la mise à mort. Mayom jora, verser le sang, est essentiel au sacrifice.

Tuer la victime n'est qu'une technique qui peut être désignée par différentes expressions selon la méthode utilisée. Ceci trouve sa vêrification dans le fait que le prêtre santal ne met pas toujours luimême à mort la victime et fait souvent appel à un assistant, mais il est néanmoins considéré comme le sacrificateur parce que, d'une part,il consacre la victime et que, d'autre part, il répand le sang de celle-ci de gauche à droite dans le diagrame rituel.

Les différents types de mise à mort font référence à des rites très différents et correspondent également à des unitês de culte hétérogènes. Ainsi, les descendants d'un même ancêtre déchirent le cou (jabe) de leur victime; les descendants d'un groupe d'ancêtres communs, au niveau du sous-clan cette fois, écrasent la nuque de leurs victimes (boucs, chèvres, porcs, buffles parfois), il s'agit de sacrifices plus occasionnels offerts à la divinité de lieu d'un sous-clan. Cette façon de mettre à mort, kutom dangra, concerne davantage les boeufs, vaches et buffles sacrifiês aux divinités du groupe local de sous-clan.

-- mayam jora : verser le sang; ceci se fait de gauche à droite, soit du nord au sud. Le sang d'un animal consacré à une divinité n'est 
pas immédiatement versé dans les cases du diagramme où "se tiennent" les autres divinités qui attendent leur tour. La couleur différente des victimes vient ici éviter tout risque de confusion. Lorsqu'une même divinité reçoit le sang de plusieurs animaux, le prêtre prend soin de ne pas mêler le sang des différentes victimes, qui est alors versé sur des tas de riz différents. Par 1'effet de la libation de sang, il n'y a plus que des différences d'une divinité à l'autre, écarts marqués par les intervalles qui séparent les tas de riz; les figures rituelles reprêsentent d'une manière formelle la hiérarchie entre les divinités qui se trouve abolie par 1 'acte du sacrifice. -- jom $\dot{n} u$ : manger et boire ; il s'agit de la consommation des victimes du sacrifice qui, dans le cas des rites publics, a lieu dans le bosquet sacré. Les têtes des animaux sont rôties et exclusivement consommées par les prêtres; manger la tête de l'animal sacrificiel, c'est se mettre en contact étroit avec la victime et, par là-même, avec la divinité ; c'est également consommer la partie de l'animal sur laquelle a porté le geste de mise à mort. C'est par le cou que "l'on ouvre le chemin du sacrifice" ; ce chemin est celui du sang qui véhicule le riz sacrificiel aux divinités. Si une femme, par erreur, consommait la tête d'une victime, elle deviendrait possédée par la divinité à laquelle ce sacrifice était destinê et sombrerait dans la folie (konko). La transgression d'un tel interdit non seulement compromettrait le bénéfice attendu du sacrifice, mais encore bouleverserait l'ordre sur lequel repose le sacrifice santal, ordre dont le naeke, prêtre du village, est le garant vis-à-vis du couple ancestral.

A l'inverse de leurs voisins hindous de la sociêté des castes, les Santal ne considèrent pas que la consommation du repas sacrificiel soit équivalente à celle des restes des dieux. Ils insistent au contraire sur le partage : les dieux reçoivent le sang, les hommes consomment la chair.

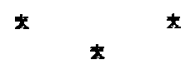

L'opposition des sacrifices et des offrandes végétales propre à 1 'Inde hindoue structure en partie le sacrifice santal. Chez les Santal 
les divinités d'origine hindoue, lorsqu'elles sont végétariennes, sont liées au destin personnel de l'individu, le sacrifice devient alors modalité de l'action humaine comme dans la société des castes.

Les sacrifices d'animaux suivis de libations de sang humain sont liês à des pratiques d'exorcisme qui visent à détruire les divinités ou leurs substituts matérialisês (20) ; ils caractérisent les rites d'où sont exclues les divinités hindoues.

Le langage du sacrifice êvoque la mort ; à 1'inverse des hindous, les Santal ne se sentent aucune dette vis-à-vis des divinitês mais savent qu'ils doivent se libérer des dettes ponctuelles qu'ils contractent lorsqu'ils font aux bonga des promesses de sacrifice. Les promesses de sacrifice manifestent à la fois le "doute" (dokha) que les Santal entretiennent envers les divinités et une situation économique prêcaire où les bonga deviennent les crêanciers des humains. Loin d'écouler dans le sacrifice un surplus, les Santal s'endettent pour le sacrifice et accumulent au niveau de la complexité des prescriptions ce qu'ils ne peuvent accumuler sous forme de biens. La justification de la complexité du sacrifice, c'est ici la stratégie dont on use envers les bonga ; ces derniers n'existent, disent les prêtres santal, que par le sacrifice; les invocations s'adressent souvent à l'agent supposé du mal qui trouble les humains, dont l'existence n'est pas encore reconnue, "vous qui n'existez pas", "celui d'entre vous qui". Le sacrifice devient alors un dispositif à ênoncer un discours où 1 'existence des divinités est tour à tour affirmẻe et niêe.

20. Dans une écorce d'arbre, par exemple. 